\title{
Use of electronic medical records for improving accurate coding of dermatology procedures
}

\author{
Authors: Saman Zaman, Lubiana Shabeer, Andreea Anton and Iaisha Ali
}

\begin{abstract}
Aims
Coding of dermatology surgery procedures allows the trust to be paid for these services. This is dependent on accurate documentation from the operating doctor.

Historically, this relied on a laborious process of analysing paper notes that were often incomplete, hard to decipher or sometimes missing altogether. This led to low reimbursement for activities carried out by our department and we sought to utilise the new electronic medical records (EMR), Cerner, system to be able to capture our procedure activity. The idea was to enter procedure coding directly on Cerner by the operator, using options to select from the Office of Population Censuses and Surveys Classification of Surgical Operations and Procedures (OPCS-4).

Our aim was to investigate the accuracy of this electronic coding at source method, and to make improvements if needed.
\end{abstract}

\section{Methods}

We completed an initial audit of the coding entered for biopsy lists between January and February 2017. We compared data from the procedure documentation notes and the clinic's procedure logbook with the information entered in the EMR's 'procedure codes' section.

To address the shortfall in accurate coding, a trust-wide departmental educational programme was initiated to explain coding practice and its recording using EMR. We recruited the help of champions in the form of lead consultants. We also gave individual tutorials to operators whose lists were being especially poorly coded. We found that locum doctor lists suffered the lowest coding accuracy, and thus updated their induction to include this information.

One month after the programme, we conducted a re-audit of the coding accuracy using a retrospective observational method as before.

Results

We found shortfalls in accurate coding practice across the trust's dermatology department, but also a wide discrepancy between the sites, particularly due to entire regular lists being uncoded.

\begin{tabular}{llll}
\multicolumn{2}{l}{ Table 1. Initial audit } \\
& Cases, $\mathbf{n}$ & Coded, $\mathbf{n}$ & Percentage, \% \\
CX & 86 & 57 & $66.3 \%$ \\
SMH & 55 & 49 & $89.1 \%$ \\
$\mathrm{HH}$ & 33 & 29 & $87.9 \%$ \\
Trust total & 174 & 135 & $77.6 \%$
\end{tabular}

\begin{tabular}{llll}
\multicolumn{4}{c}{ Table 2. Re-audit post-intervention } \\
& Cases, $\mathbf{n}$ & Coded, $\mathbf{n}$ & Percentage, $\%$ \\
CX & 116 & 107 & $92.2 \%$ \\
SMH & 81 & 69 & $85.2 \%$ \\
$\mathrm{HH}$ & 70 & 63 & $90.0 \%$ \\
Trust total & 267 & 239 & $89.5 \%$
\end{tabular}

Through our intervention, we found an overall $12 \%$ improvement in correct coding in dermatology across the trust (77.6\% to $89.5 \%$ ), and the lowest attaining site improved by $26 \%$ from $66.3 \%$ to $92.2 \%$.

\section{Conclusion}

Clinical coding is a poorly understood concept by clinical staff, however the implications of the quality of documentation by front-line staff directly affects the financial remuneration to the trust. Our work shows how with improved departmental education, operators can be supported to increase accuracy of coding practice.

\section{Conflict of interest statement}

None declared. 